Pacific Journal of Mathematics

aRGALIS 


\title{
NONLINEAR INTEGRAL EQUATIONS AND PRODUCT INTEGRALS
}

\author{
Alvin J. KaY
}

B. W. Helton has studied linear equations of the form

$$
f(x)=f(a)+(R L) \int_{x}^{a}(K f+M f)
$$

this paper extends some of his results to a nonlinear setting. Let $S$ be a linearly ordered set, $\{G,+,\|\|\}$ a complete normed abelian group, $H$ the set of functions from $G$ to $G$ that take 0 to $0, \mathscr{O A}$ and $O M$ classes of functions from $S X S$ to $H$ that are order-additive and order-multiplicative respectively and satisfy a Lipschitz-type condition, and $\mathscr{E}$ be J. S. Mac Nerney's reversible mapping from $\mathscr{O A}$ onto $\mathscr{O} M$. If $\{V, W\}$ is in $\mathscr{E}$, we show the collection of all functions that are differentially equivalent to $V$ is the same as the collection of functions that are differentially equivalent to $W-1$. This analysis is used to prove existence theorems for product integrals which we show solve (1).

1. Introduction. In his 1966 paper, Integral Equations and Product Integrals [2], B. W. Helton obtained product integral solutions of linear integral equations of the form (1) where the integration is directed along intervals in some linearly ordered system, the functions involved have their values in a complete normed ring, and the right-left integral is of the subdivision-refinement type.

We extend some of these results to the nonlinear setting developed by J. S. Mac Nerney in [7]. As in [7], $S$ denotes some nondegenerate set, with linear ( $\leqq$ ) ordering $\mathcal{O} ;\{G,+,\|\|\}$ denotes a complete normed abelian group with zero element 0 , and $H$ denotes the class of all functions from $G$ to $G$ to which $\{0,0\}$ belongs, with identity function 1. $\mathscr{O} \mathscr{A}^{+}$denotes the class of all $\mathcal{O}$-additive functions from $S X S$ to the set of nonnegative real numbers, and $\mathrm{OM}^{+}$denotes the class of all $\mathcal{O}$ - multiplicative functions from $S X S$ to the set of real numbers not less than 1.

The class $\mathscr{O A}$ consists of all functions $V$ from $S X S$ to $H$ such that

(i) $V$ is $\mathcal{O}$-additive in the sense that, for each $\{x, z, P\}$ in $S X S X G$, if $\{x, y, z\}$ is an $\mathcal{O}$-subdivision of $\{x, z\}$ then

$$
V(x, y) P+V(y, z) P=V(x, z) P, \quad \text { and }
$$


(ii) there is a member $\alpha$ in $\mathscr{O A} \mathscr{A}^{+}$such that if $\{x, y\}$ is in $S X S$ and $\{P, Q\}$ is in $G X G$ then

$$
\|V(x, y) P-V(x, y) Q\| \leqq \alpha(x, y)\|P-Q\| .
$$

The class $\mathcal{O} \mathcal{M}$ consists of all functions $W$ from $S X S$ to $H$ such that

(i) $W$ is $\mathscr{O}$-multiplicative in the sense that, for each $\{x, z\}$ in $S X S$ and $P$ in $G$, if $\{x, y, z\}$ is an $\mathcal{O}$-subdivision of $\{x, z\}$ then

$$
W(x, y) W(y, z) P=W(x, z) P, \quad \text { and }
$$

(ii) there is a member $\mu$ of $\mathscr{O} \mathcal{M}^{+}$such that if $\{x, y\}$ is in $S X S$ and $\{P, Q\}$ is in $G X G$ then

$$
\|[W(x, y)-1] P-[W(x, y)-1] Q\| \leqq[\mu(x, y)-1]\|P-Q\| .
$$

In [7], Mac Nerney establishes that there is a reversible function $\mathscr{E}$ from $\mathscr{O A}$ onto $O M$ such that if $V$ is in $\mathscr{O A}$ and $W=\mathscr{E}(V)$ then, for $\{x, y, P\}$ in $S X S X G$,

$$
W(x, y) P={ }_{x} \prod^{y}[1+V] P \text { and } V(x, y) P={ }_{x} \Sigma^{y}[W-1] P .
$$

If $\{V, W\}$ is in $\mathscr{E}$, we show the collection of functions that are differentially equivalent to $V$ is the same as the collection of functions that are differentially equivalent to $W-1$ (i.e., functions $M$ and $N$ from $S X S$ to $H$ are differentially equivalent only in case there is a function $k$ from $S X S$ to the real numbers such that ${ }_{x} \Sigma^{y} k=0$ and $\|M(x, y) P-N(x, y) P\| \leqq k(x, y)\|P\|$ for each $\{x, y, P\}$ in $S X S X G$ [6]). This analysis is used to prove existence theorems for product integrals of the form

$$
W(x, y) P={ }_{x} \prod^{y}[1-M]^{-1}[1+K] P,
$$

where $\{x, y, P\}$ is in $S X S X G$ and there is a $\left\{V_{1}, V_{2}\right\}$ in $\mathscr{O A X O A A}$ such that $K$ and $M$ are differentially equivalent to $V_{1}$ and $V_{2}$ respectively. Product integrals of this form were introduced in the linear case by Helton in [2]. In addition, we show that if $V(x, y) P=$ ${ }_{x} \Sigma^{y}\left\{[1-M]^{-1}[1+K]-1\right\} P$ for each $\{x, y, P\}$ in $S X S X G$, then $\{V, W\}$ is in $\mathscr{E}$. Finally we show if $f$ is a function from $S$ to $G$ that is of bounded variation on each $\mathcal{O}$-interval of $S$ then $W(x, a) f(a)$ solves (1) and as in $[7, \S 3]$ it is shown that the theory of the seemingly more general equation

$$
u(x)=P_{1}+(R L) \int_{c}^{x}(K u+M u)+V(x, c) P_{2}
$$

is subsumed in this treatment. 
In [7, p. 624] Professor Mac Nerney defines sum and product integrals in this setting. We indicate the definitions: if $g$ is a function from $S X S$ to $G, h$ is a function from $S X S$ to $H$ and $\{x, y, P\}$ is in $S X S X G,{ }_{x} \sum^{y} g \sim \sum_{1}^{n} g\left(t_{j-1}, t_{j}\right)$ and ${ }_{x} \prod^{y}[h] P \sim\left\{\prod_{1}^{n} h\left(t_{j-1}, t_{j}\right)\right\} P$ (functional composite) where $\left\{t_{j}\right\}_{0}^{n}$ is an $\mathcal{O}$-subdivision of $\{x, y\}$.

Let $\Phi$ denote a function from $O \mathscr{A}$ such that if $V$ is in $O \mathscr{A}$ then $\Phi(V)$ is the set to which $K$ belongs only in case $K$ is differentially equivalent to $V$.

Let $\psi$ denote a function from $O M$ such that if $W$ is in $O M$ then $\psi(W)$ is the set to which $K$ belongs only in case $K$ is differentially equivalent to $W-1$.

Remark. In [2, p. 299] Professor Helton defines function classes $O A^{0}, O M^{0}$ and $O B^{0}$. In the linear case, our $\Phi(O A A)$ includes the common part of $O A^{\circ}$ and $O B^{\circ}$ and $\psi(O M)$ includes the common part of $O M^{0}$ and $O B^{0}$.

2. $\psi[\mathscr{E}]=\Phi$. In this section we prove two theorems that will be used in the proofs of later theorems. In the first theorem we prove that if $K$ is in $\psi(O M)$ then the sum and product integrals of $K$ exist and in the second theorem we prove that if $\{V, W\}$ is in $\mathscr{E}$, the collection of functions which are differentially equivalent to $V$ is the same as the collection of functions which are differentially equivalent to $W-1$.

Theorem 2.1. If $\{V, W\}$ is in $\mathscr{E}$ and $K$ is in $\psi(W)$ then

(1) $W(x, y) P={ }_{x} \Pi^{y}[1+V] P={ }_{x} \Pi^{y}[1+K] P$ for every $\{x, y, P\}$ in $S X S X G$, and

(2) $V(x, y) P={ }_{x} \Sigma^{y}[W-1] P={ }_{x} \Sigma^{y} K P$ for every $\{x, y, P\}$ in $S X S X G$.

Proof. (1) Let $W$ be in $O \mathcal{M}$ and $K$ in $\psi(W), k$ be a function from $S X S$ to the real numbers such that for $\{x, y, P\}$ in $S X S X G$

$$
\|K(x, y) P-[W(x, y)-1] P\| \leqq k(x, y)\|P\|
$$

and ${ }_{x} \Sigma^{y} k=o$, and $\mu$ be a member of $O \mathcal{M}^{+}$such that for each $\{x, y\}$ in $S X S$ and $\{P, Q\}$ in $G X G$

$$
\|[W(x, y)-1] P-[W(x, y)-1] Q\| \leqq[\mu(x, y)-1]\|P-Q\| .
$$

Suppose $c$ is a positive number and $\{x, y, P\}$ is in $S X S X G$. There is an $\mathcal{O}$-subdivision $s$ of $\{x, y\}$ such that if $\left\{t_{j}\right\}_{0}^{n}$ is a refinement of $s$ then $\sum_{1}^{n} k\left(t_{j-1}, t_{j}\right)<c / 2 \mu(x, y)^{2}$ and $\operatorname{Exp}\left[\Sigma_{1}^{n} k\left(t_{j-1}, t_{j}\right)\right]<2$. By Lemma 1.2 [7, p. 623] 


$$
\begin{aligned}
\| \prod^{n} & {\left[1+K\left(t_{j-1}, t_{j}\right)\right] P-{ }_{1} \prod^{n} W\left(t_{j-1}, t_{j}\right) P \| } \\
& \leqq\left\{\prod_{1}^{n}\left[\mu\left(t_{j-1}, t_{j}\right)+k\left(t_{j-1}, t_{j}\right)\right]-\prod^{n} \mu\left(t_{j-1}, t_{j}\right)\right\}\|P\| \\
& =\sum_{j=1}^{n} \prod_{i=1}^{j-1} \mu\left(t_{i-1}, t_{i}\right) k\left(t_{j-1}, t_{j}\right) \prod_{q=j+1}^{n}\left[\mu\left(t_{q-1}, t_{q}\right)+k\left(t_{q-1}, t_{q}\right)\right]\|P\| \\
& \leqq \mu(x, y)^{2} \operatorname{Exp}\left[\sum_{j=1}^{n} k\left(t_{j-1}, t_{j}\right)\right] \sum_{j=1}^{n} k\left(t_{j-1}, t_{j}\right)\|P\|<c\|P\| .
\end{aligned}
$$

(2) For each $\mathcal{O}$ - subdivision $\left\{t_{j}\right\}_{0}^{n}$ of $\{x, y\}$ in $S X S$

$$
\begin{aligned}
& \left\|V(x, y) P-\sum_{t}^{n} K\left(t_{j-1}, t_{j}\right) P\right\| \\
& \leqq \sum_{1}^{n}\left\|\left[W\left(t_{j-1}, t_{j}\right)-1\right] P-K\left(t_{j-1}, t_{j}\right) P\right\| \\
& \quad+\left\|\sum_{t}[W-1] P-V(x, y) P\right\| \\
& \leqq \\
& \left.\quad \sum_{1}^{n} k\left(t_{j-1}, t_{j}\right)+\sum_{t}[\mu-1]-\alpha(x, y)\right\}\|P\| .
\end{aligned}
$$

Since ${ }_{x} \Sigma^{y} k+{ }_{x} \Sigma^{y}(\mu-1)-\alpha(x, y)=0$ the proof is complete.

REMARK. The proof of the following theorem is similar to the proof of Theorem $3.4[2$, p. 301] of which this theorem is an extension.

THEOREM 2.2. $\psi[[\mathscr{E}]=\Phi$.

Proof. Part I. Let $V$ be in $\mathscr{O A}$ and $\mathscr{E}(V)=W$ and $K$ be in $\psi(W)$; there is a $\mu$ in $\mathscr{O} \mathcal{M}^{+}$such that for each $\{x, y\}$ in $S X S$ and $\{P, Q\}$ in GXG

$$
\|\{W(x, y)-1\} P-\{W(x, y)-1\} Q\| \leqq[\mu(x, y)-1]\|P-Q\| .
$$

Also there is a function $k$ from $S X S$ to the real numbers such that ${ }_{x} \Sigma^{y} k=0$ and

$$
\|\{1+K(x, y)\} P-W(x, y) P\| \leqq k(x, y)\|P\| .
$$


By Corollary $1.1[7$, p. 626]

$$
\begin{aligned}
& \left\{[\mu(x, y)-1]-{ }_{x} \sum^{y}[\mu-1]\right\}\|P\| \\
& \quad \geqq\left\|[W(x, y)-1] P-{ }_{x} \sum^{y}[W-1] P\right\| \\
& \quad \geqq\left\|K(x, y) P-{ }_{x} \sum^{y}[W-1] P\right\|-\|[1+K(x, y)] P-W(x, y) P\| ;
\end{aligned}
$$

hence

$$
\|K(x, y) P-V(x, y) P\| \leqq\left\{[\mu(x, y)-1]-{ }_{x} \sum^{y}[\mu-1]+k(x, y)\right\}\|P\|
$$

so $K$ is in $\Phi(V)$.

Part 2. Let $K$ be in $\Phi(V)$; there is an $\alpha$ in $\mathscr{O A} A^{+}$such that if $\{x, y\}$ is in $S X S$ and $\{P, Q\}$ is in $G X G$ then

$$
\|V(x, y) P-V(x, y) Q\| \leqq \alpha(x, y)\|P-Q\|
$$

and there is a function $h$ from $S X S$ to the real numbers such that $\|V(x, y) P-K(x, y) P\| \leqq h(x, y)\|P\|$ and ${ }_{x} \Sigma^{y} h=0$. By Corollary 1.1 $[7$, p. 626]

$$
\begin{aligned}
& \|[1+K(x, y)] P-W(x, y) P\| \\
\leqq & \left\|[1+V(x, y)] P-{ }_{x} \Pi^{y}[1+V] P\right\|+\|V(x, y) P-K(x, y) P\| \\
\leqq & {\left[{ }_{x} \prod^{y}[1+\alpha]-\alpha(x, y)-1+h(x, y)\right]\|P\| ; \text { therefore } K \text { is in } \psi(\mathscr{E}(V)) . }
\end{aligned}
$$

3. Existence theorems. In this section we will prove that if each of $K$ and $M$ is in $\Phi(\mathscr{O A})$ and $[1-M(x, y)]^{-1} P$ exists and is bounded sufficiently there is a member $V$ of $\mathscr{O A}$ such that

$$
[1+K][1-M]^{-1}-1
$$

is in $\Phi(V)$; hence ${ }_{x} \Pi^{y}[1+K][1-M]^{-1} P={ }_{x} \Pi^{y}[1+V] P$ for every $\{x, y, P\}$ in $S X S X G$. This extends existence theorems proven by J. $S$. Mac Nerney [7], B. W. Helton [3], J. V. Herod [5] and J. C. Helton [4].

THEOREM 3.0. If each of $\alpha_{1}$ and $\alpha_{2}$ is in $\mathscr{O A} A^{+}$and $\alpha_{2}(x, y)<1$ for each $\{x, y\}$ in $S X S$, then

(1) $\alpha(x, y)={ }_{x} \Sigma^{y}\left\{\left[1+\alpha_{1}\right]\left[1-\alpha_{2}\right]^{-1}-1\right\}$ exists for each $\{x, y\}$ in $S X S$ and $\alpha$ is in $\mathscr{O A} \mathscr{A}^{+}$; 
(2) $\mu(x, y)={ }_{x} \Pi^{y}\left[1+\alpha_{1}\right]\left[1-\alpha_{2}\right]^{-1}$ exists for each $\{x, y\}$ in $S X S$ and $\mu$ is in $\mathcal{O M}^{+}$; and

(3) $\mu(x, y)={ }_{x} \Pi^{y}\{1+\alpha\}$ for each $\{x, y\}$ in $S X S$.

Proof. Let $\beta=\left[1+\alpha_{1}\right]\left[1-\alpha_{2}\right]^{-1}-1$; if $\{r, s, t\}$ is an $\mathcal{O}$-subdivision of $\{r, t\}$ in $S X S$,

$$
\beta(r, t) \geqq \beta(r, s)+\beta(s, t) \geqq 0 .
$$

Hence $\alpha(x, y)={ }_{x} \Sigma^{y} \beta=$ G.L.B. $\Sigma_{t} \beta \geqq 0$ for all $\mathcal{O}$-subdivisions $t$ of $\{x, y\}$ in $S X S . \quad \beta$ is in $\Phi(\alpha)$ and from Theorem $2.2 \beta$ is in $\psi(\mathscr{E}(\alpha))$. Hence from Theorem $2.1 \mu(x, y)={ }_{x} \Pi^{y}[1+\beta]={ }_{x} \Pi^{y}[1+\alpha]$ for all $\{x, y\}$ in $S X S$, and the proof is complete.

Remark. As noted by Herod [5, p. 188] and proved by Neuberger [8, p. 101], if $T$ is in $H$ and $0<t<1$ and $\|T P-T Q\| \leqq t\|P-Q\|$ for all $\{P, Q\}$ in $G X G$ then $(1-T)^{-1}$ is in $H,(1-T)^{-1}=1+T(1-T)^{-1}$, and for each such $\{P, Q\}\left\|(1-T)^{-1} P-(1-T)^{-1} Q\right\| \leqq(1-t)^{-1}\|P-Q\|$. These and closely related inequalities are used in the sequel, usually without explicit reference.

THEOREM 3.1. If each of $V_{1}$ and $V_{2}$ is in $O \mathscr{A}$ and each of $\alpha_{1}$ and $\alpha_{2}$ is in $\mathscr{O A}^{+}$such that for $\{x, y\}$ in $S X S$ and $\{P, Q\}$ in $G X G, \alpha_{2}(x, y)<1$,

then

$$
\begin{aligned}
& \left\|V_{1}(x, y) P-V_{1}(x, y) Q\right\| \leqq \alpha_{1}(x, y)\|P-Q\| \quad \text { and } \\
& \left\|V_{2}(x, y) P-V_{2}(x, y) Q\right\| \leqq \alpha_{2}(x, y)\|P-Q\|,
\end{aligned}
$$

(1) $V(x, y) P={ }_{x} \Sigma^{y}\left\{\left[1+V_{1}\right]\left[1-V_{2}\right]^{-1}-1\right\} P$ exists for each $\{x, y, P\}$ in $S X S X G$ and $V$ is in $O A A$;

(2) $W(x, y) P={ }_{x} \Pi^{y}\left[1+V_{1}\right]\left[1-V_{2}\right]^{-1} P$ exists for each $\{x, y, P\}$ in $S X S X G$ and $W$ is in $O M$; and

(3) $\{V, W\}$ is in $\mathscr{E}$.

Proof. (1) Note that $\left[1+V_{1}\right]\left[1-V_{2}\right]^{-1}-1=\left[V_{1}+V_{2}\right]\left[1-V_{2}\right]^{-1}$ and if $\{x, y, P\}$ is in $S X S X G$ and $\{x, s, t, y\}$ is an $\mathcal{O}$ - subdivision of $\{x, y\}$ then

$$
\begin{aligned}
\|[1- & \left.V_{2}(x, y)\right]^{-1} P-\left[1-V_{2}(s, t)\right]^{-1} P \| \\
= & \| V_{2}(x, y)\left[1-V_{2}(x, y)\right]^{-1} P-V_{2}(s, t)\left[1-V_{2}(s, t)\right]^{-1} P \\
& \pm V_{2}(x, y)\left[1-V_{2}(s, t)\right]^{-1} P \| \\
\leqq & \alpha_{2}(x, y)\left\|\left[1-V_{2}(x, y)\right]^{-1} P-\left[1-V_{2}(s, t)\right]^{-1} P\right\| \\
& +\left[\alpha_{2}(x, y)-\alpha_{2}(s, t)\right]\left[1-\alpha_{2}(s, t)\right]^{-1}\|P\| \\
\leqq & \left\{\left[1-\alpha_{2}(x, y)\right]^{-1}-\left[1-\alpha_{2}(s, t)\right]^{-1}\right\}\|P\| .
\end{aligned}
$$


For each $\{x, y, P\}$ in $S X S X G$ and $\mathcal{O}$-subdivision $\left\{t_{j}\right\}_{0}^{n}$ of $\{x, y\}$

$$
\begin{aligned}
&\left\|\left[V_{1}(x, y)+V_{2}(x, y)\right]\left[1-V_{2}(x, y)\right]^{-1} P-\sum_{t}\left[V_{1}+V_{2}\right] \times\left[1-V_{2}\right]^{-1} P\right\| \\
&=\left\|\left\{\sum_{t}\left[V_{1}+V_{2}\right]\right\}\left[1-V_{2}(x, y)\right]^{-1} P-\sum_{t}\left[V_{1}+V_{2}\right]\left[1-V_{2}\right]^{-1} P\right\| \\
& \leqq \sum_{1}^{n}\left[\alpha_{1}\left(t_{j-1}, t_{j}\right)+\alpha_{2}\left(t_{j-1}, t_{j}\right)\right]\left\{\left[1-\alpha_{2}(x, y)\right]^{-1}\right. \\
&\left.-\left[1-\alpha_{2}\left(t_{j-1}, t_{j}\right)\right]^{-1}\right\}\|P\| \\
&=\left\{\left[\alpha_{1}(x, y)+\alpha_{2}(x, y)\right]\left[1-\alpha_{2}(x, y)\right]^{-1}-\sum_{t}\left[\alpha_{1}+\alpha_{2}\right] \times\left[1-\alpha_{2}\right]^{-1}\right\}\|P\| .
\end{aligned}
$$

If follows that if $s$ is a refinement of $t$

$$
\begin{aligned}
\| \sum_{s}\left[V_{1}\right. & \left.+V_{2}\right]\left[1-V_{2}\right]^{-1} P-\sum_{t}\left[V_{1}+V_{2}\right]\left[1-V_{2}\right]^{-1} P \| \\
& \leqq\left\{\sum_{s}\left[\alpha_{1}+\alpha_{2}\right]\left[1-\alpha_{2}\right]^{-1}-\sum_{t}\left[\alpha_{1}+\alpha_{2}\right]\left[1-\alpha_{2}\right]^{-1}\right\}\|P\| .
\end{aligned}
$$

Hence, by the completeness of $\{G,+,\|\|\}$ and Theorem 3.0 $V(x, y) P={ }_{x} \Sigma^{y}\left[V_{1}+V_{2}\right]\left[1-V_{2}\right]^{-1} P$ exists. For each $\{x, y\}$ in $S X S$ and $\{P, Q\}$ in $G X G\|V(x, y) P-V(x, y) Q\| \leqq \alpha(x, y)\|P-Q\|$ where $\alpha$ is defined as in Theorem 3.0. Therefore $V$ is in $\mathscr{O A}$ and, with $\beta$ as in the proof of Theorem 3.0, considerations of $\beta-\alpha$ may be seen to show that $\left[1+V_{1}\right]\left[1-V_{2}\right]^{-1}-1$ is in $\Phi(V)$. (2) and (3) follow from (1) and Theorems 2.1 and 2.2 .

THEOREM 3.2. If each of $V_{1}$ and $V_{2}$ is in $\mathcal{O A}$, and each of $\alpha_{1}$ and $\alpha_{2}$ is in $\mathscr{O A}^{+}$such that for each $\{x, y\}$ in $S X S$ and $\{P, Q\}$ in $G X G$ $\alpha_{2}(x, y)<1$,

$$
\begin{aligned}
& \left\|V_{1}(x, y) P-V_{1}(x, y) Q\right\| \leqq \alpha_{1}(x, y)\|P-Q\|, \text { and } \\
& \left\|V_{2}(x, y) P-V_{2}(x, y) Q\right\| \leqq \alpha_{2}(x, y)\|P-Q\|,
\end{aligned}
$$

then

(1) $V(x, y) P={ }_{x} \Sigma^{y}\left\{\left[1-V_{2}\right]^{-1}\left[1+V_{1}\right]-1\right\} P$ exists for each $\{x, y, P\}$ in $S X S X G$, and $V$ is in $\mathscr{O A}$;

(2) $W(x, y) P={ }_{x} \Pi^{y}\left[1-V_{2}\right]^{-1}\left[1+V_{1}\right] P$ exists for each $\{x, y, P\}$ in $S X S X G$ and $W$ is in $O M$; and

(3) $\{V, W\}$ is in $\mathscr{E}$. 
Proof. (1) Note that

$$
\left[1-V_{2}\right]^{-1}\left[1+V_{1}\right]-1=V_{1}+V_{2}\left[1-V_{2}\right]^{-1}\left[1+V_{1}\right] \text { and if }\{x, y, P\}
$$

is in $S X S X G$ and $\{x, s, t, y\}$ is an $\mathcal{O}$-subdivision of $\{x, y\}$ then

$$
\begin{aligned}
\|[1- & \left.V_{2}(x, y)\right]^{-1}\left[1+V_{1}(x, y)\right] P-\left[1-V_{2}(s, t)\right]^{-1}\left[1+V_{1}(s, t)\right] P \| \\
= & \| V_{1}(x, y) P-V_{1}(s, t) P+V_{2}(x, y)\left[1-V_{2}(x, y)\right]^{-1}\left[1+V_{1}(x, y)\right] P \\
& -V_{2}(s, t)\left[1-V_{2}(s, t)\right]^{-1}\left[1+V_{1}(s, t)\right] P \\
& \pm V_{2}(x, y)\left[1-V_{2}(s, t)\right]^{-1}\left[1+V_{1}(s, t)\right] \| \\
\leqq & {\left[\alpha_{1}(x, y)-\alpha_{1}(s, t)\right]\|P\|+\alpha_{2}(x, y) \|\left[1-V_{2}(x, y)\right]^{-1}\left[1+V_{1}(x, y)\right] P } \\
& -\left[1-V_{2}(s, t)\right]^{-1}\left[1+V_{1}(s, t)\right] P \| \\
& +\left[\alpha_{2}(x, y)-\alpha_{2}(s, t)\right]\left[1-\alpha_{2}(s, t)\right]^{-1}\left[1+\alpha_{1}(s, t)\right]\|P\| \\
\leqq & {\left.\left[1-\alpha_{2}(x, y)\right]^{-1}\left[1+\alpha_{1}(x, y)\right]-\left[1-\alpha_{2}(s, t)\right]^{-1}\left[1+\alpha_{1}(s, t)\right]\right\}\|P\| . }
\end{aligned}
$$

For each $\{x, y, P\}$ in $S X S X G$ and $\mathcal{O}$-subdivision $\left\{t_{j}\right\}_{0}^{n}$ of $\{x, y\}$

$$
\begin{aligned}
& \| V_{1}(x, y) P+V_{2}(x, y)\left[1-V_{2}(x, y)\right]^{-1}\left[1+V_{1}(x, y)\right] P \\
&-\sum_{t}\left\{V_{1}+V_{2}\left[1-V_{2}\right]^{-1}\left[1+V_{1}\right]\right\} P \| \\
&= \|\left\{\sum_{t} V_{2}\right\}\left[1-V_{2}(x, y)\right]^{-1}\left[1+V_{1}(x, y)\right] P \\
& \leqq \sum_{1}^{n} \alpha_{2}\left(t_{j-1}, t_{j}\right)\left\{\left[1-\alpha_{2}(x, y)\right]^{-1}\left[1+\alpha_{1}(x, y)\right]\right. \\
&-\left[1-\alpha_{2}\left(t_{j-1}, t_{j}\right)\right]^{-1}\left[1+V_{1}\right] P \| \\
&=\left.\left\{\left[1-\alpha_{2}(x, y)\right]^{-1}\left[1+\alpha_{1-1}, t_{j}\right)\right]\right\}\|P\|
\end{aligned}
$$

The rest of the proof is identical to the proof of Theorem 3.1.

Theorem 3.3. Suppose

(1) each of $V_{1}$ and $V_{2}$ is in $\mathscr{O A}$, and each of $\alpha_{1}$ and $\alpha_{2}$ is in $\mathscr{O A}+$ such that for each $\{x, y, P, Q\}$ in $S X S X G X G$

$$
\begin{aligned}
& \left\|V_{1}(x, y) P-V_{1}(x, y) Q\right\| \leqq \alpha_{1}(x, y)\|P-Q\| \text { and } \\
& \left\|V_{2}(x, y) P-V_{2}(x, y) Q\right\| \leqq \alpha_{2}(x, y)\|P-Q\| ;
\end{aligned}
$$

(2) $K$ is in $\Phi\left(V_{1}\right)$ and $M$ is in $\Phi\left(V_{2}\right)$ and each of $h$ and $k$ is a function from $S X S$ to the real numbers such that for each $\{x, y, P\}$ in 
$S X S X G,{ }_{x} \Sigma^{y} k=0,\left\|K(x, y) P-V_{1}(x, y) P\right\| \leqq h(x, y)\|P\|,{ }_{x} \Sigma^{y} h=0$ and $\left\|M(x, y) P-V_{2}(x, y) P\right\| \leqq h(x, y)\|P\|$;

(3) there is a number $a<1$ such that for each $\{x, y\}$ in $S X S$ $\alpha_{2}(x, y)+h(x, y) \leqq a ;$ and

(4) $\beta=\left[1-\alpha_{2}\right]^{-1}\left[1+\alpha_{1}\right]$ and $\gamma=\left[1-\alpha_{2}-h\right]^{-1}\left[1+\alpha_{1}+k\right]$.

Conclusion :

(1) $\left\|\left[1-V_{2}(x, y)\right]^{-1}\left[1+V_{1}(x, y)\right] P-\left[1-V_{2}(x, y)\right]^{-1}\left[1+V_{1}(x, y)\right] Q\right\|$ $\leqq\left[1-\alpha_{2}(x, y)\right]^{-1}\left[1+\alpha_{1}(x, y)\right]\|P-Q\|$ for every $\{x, y\}$ in $S X S$ and $\{P, Q\}$ in $G X G$.

(2) $\left\|[1-M(x, y)]^{-1}[1+K(x, y)] P-\left[1-V_{2}(x, y)\right]^{-1}\left[1+V_{1}(x, y)\right] P\right\|$ $\leqq[\gamma(x, y)-\beta(x, y)]\|P\|$ for every $\{x, y\}$ in $S X S$ and $P$ in $G$.

(3) ${ }_{x} \Pi^{y}[1-M]^{-1}[1+K] P={ }_{x} \Pi^{y}\left[1-V_{2}\right]^{-1}\left[1+V_{1}\right] P$ for every $\{x, y, P\}$ in $S X S X G$.

Proof. Let $\{x, y\}$ be in $S X S,\{P, Q\}$ be in $G X G$ and $A=$ $\left[1-V_{2}(x, y)\right]^{-1}\left[1+V_{1}(x, y)\right]$. First note that $A=1+V_{1}(x, y)+$ $V_{2}(x, y) A$.

$$
\|\mathrm{AP}-\mathrm{AQ}\| \leqq\left[1+\alpha_{1}(\mathrm{x}, \mathrm{y})\right]\|\mathrm{P}-\mathrm{Q}\|+\alpha_{2}(\mathrm{x}, \mathrm{y})\|\mathrm{AP}-\mathrm{AQ}\| ;
$$

and assertion (1) follows. Let $B=[1-M(x, y)]^{-1}[1+K(x, y)]$; $\|B P-A P\|=$

$$
\begin{aligned}
& \|[1+K(x, y)+M(x, y) B] P-\left[1+V_{1}(x, y)+V_{2}(x, y) A\right] P \\
& \pm V_{2}(x, y) B P \| \\
& \leqq k(x, y)\|P\|+h(x, y)\|B P\|+\alpha_{2}(x, y)\|B P-A P\| \\
& \leqq k(x, y)\|P\|+h(x, y)\|A P\|+\left[h(x, y)+\alpha_{2}(x, y)\right]\|B P-A P\| \\
& \leqq k(x, y)\|P\|+h(x, y)\left[1+\alpha_{1}(x, y)\right]\left[1-\alpha_{2}(x, y)\right]^{-1}\|P\| \\
& +\left[h(x, y)+\alpha_{2}(x, y)\right]\|B P-A P\|
\end{aligned}
$$

which, except for minor algebraic manipulation, establishes (2).

For each $\mathcal{O}$ - subdivision $t$ of $\{x, y\}$ it follows from Lemma 1.2 [7, p. 623] that

$$
\left\|\prod_{t}[1-M]^{-1}[1+K] P-\prod_{t}\left[1-V_{2}\right]^{-1}\left[1+V_{1}\right] P\right\| \leqq\left\{\prod_{t} \gamma-\prod_{t} \beta\right\}\|P\| .
$$

By Theorem $3.2[2$, p. 300] and hypothesis (3) of this theorem, there is a number $b$ such that

$$
\prod_{t} \gamma-\prod_{t} \beta \leqq b^{2} \sum_{t}(\gamma-\beta) \leqq b^{3} \sum_{t} k+b^{4}\left[1+\alpha_{1}(x, y)\right] \sum_{t} h
$$


Since ${ }_{x} \sum^{y} k=0$ and ${ }_{x} \sum^{y} h=0$ the proof is complete.

TheOREM 3.4. If $V$ is in $\mathscr{O A}, \alpha$ is in $\mathscr{O A}^{+}, \alpha(x, y)<1$

$\|V(x, y) P-V(x, y) Q\| \leqq \alpha(x, y)\|P-Q\|$ and ${ }_{x} \Sigma^{y} \alpha^{2}=0$ for each $\{x, y\}$ in $S X S$ and $\{P, Q\}$ in $G X G$, then for each $\{x, y, P\}$ in $S X S X G$

(1) ${ }_{x} \prod^{y}[1-V]^{-1} P={ }_{x} \prod^{y}[1+V] P$;

(2) ${ }_{x} \prod^{y}[1+V]^{-1} P={ }_{x} \prod^{y}[1-V] P$;

(3) $\prod_{x}^{y}\left[1-V^{2}\right] P=P$; and

(4) ${ }_{x} \prod^{y}[1+V][1-V]^{-1} P={ }_{x} \prod^{y}[1+2 V] P$.

Indication of proof of (1). For each $\{x, y, P\}$ in $S X S X G$

$$
\begin{aligned}
& \left\|[1-V(x, y)]^{-1} P-[1+V(x, y)] P\right\| \\
& \quad=\left\|V(x, y)[1-V(x, y)]^{-1} P-V(x, y) P\right\| \\
& \quad \leqq \alpha(x, y)\left\|V(x, y)[1-V(x, y)]^{-1} P\right\| \\
& \quad \leqq \alpha^{2}(x, y)[1-\alpha(x, y)]^{-1}\|P\| .
\end{aligned}
$$

Similar inequalities can be established for (2), (3) and (4).

4. The integral equations. Let each of $R$ and $L$ denote a function from $S X S$ into $S$ such that $R(x, y)=y$ and $L(x, y)=x$ for each $\{x, y\}$ in $S X S$.

Remark. This notation due to W. L. Gibson in [1] provides a more precise notation for left and right integral process than that used before. Hence

$$
(R L) \int_{x}^{y}(K f+M f) \text { becomes } \int_{x}^{y}(K f[R]+M f[L]) .
$$

As in [7] $\mathscr{F}(c, P)$ denotes the class of all functions $f$ from $S$ to $G$ such that $f(c)=P$ and there is a member $\beta$ of $O \mathscr{A}^{+}$such that $\|f(y)-f(x)\| \leqq \beta(x, y)$ for each $\{x, y\}$ in $S X S$ (i.e., $f$ is of bounded variation on each $\mathcal{O}$-interval of $S$ ).

REMARK. The construction of the proof of the next lemma is similar to that of Lemma $2.2[7$, p. 623].

\section{Lemma 1. Suppose}

(1) each of $V_{1}$ and $V_{2}$ is in $\mathscr{O A}$ and $\alpha_{1}$ and $\alpha_{2}$ is in $\mathscr{O A} \mathscr{A}^{+}$such that for each $\{x, y\}$ in $S X S$ and $\{P, Q\}$ in $G X G$ 


$$
\begin{aligned}
& \left\|V_{1}(x, y) P-V_{1}(x, y) Q\right\| \leqq \alpha_{1}(x, y)\|P-Q\| \quad \text { and } \\
& \left\|V_{2}(x, y) P-V_{2}(x, y) Q\right\| \leqq \alpha_{2}(x, y)\|P-Q\| ;
\end{aligned}
$$

(2) $f$ is in $\mathscr{F}(c, P)$; and

(3) for each $\{x, y\}$ in $S X S$

$$
C(x, y)=\int_{x}^{y}\left\{V_{1} f[R]+V_{2} f[L]\right\}-V_{1}(x, y) f(y)-V_{2}(x, y) f(x) .
$$

Conclusion. For each $\{x, y\}$ in $S X S$

$$
\sum^{y}\|C\|=0 \text {. }
$$

Proof. Let $\beta$ be in $\mathscr{O} \mathscr{A}^{+}$such that $\|d f\| \leqq \beta,\{x, y\}$ be in $S X S$ such that $\{x, y, c\}$ is an $\mathcal{O}$-subdivision of $\{x, c\}$ where $c$ is in $S$, and $\left\{t_{i}\right\}_{0}^{n}$ be an $\mathcal{O}$-subdivision of $\{x, y\}$; then

$$
\begin{aligned}
&\left\|\sum_{t}\left\{V_{1} f[R]+V_{2} f[L]\right\}-V_{1}(x, y) f(y)-V_{2}(x, y) f(x)\right\| \\
& \leqq\left\|\sum_{1}^{n} V_{2}\left(t_{i-1}, t_{i}\right) f\left(t_{i-1}\right)-\sum_{1}^{n} V_{2}\left(t_{i-1}, t_{i}\right) f(x)\right\| \\
&+\left\|\sum_{1}^{n} V_{1}\left(t_{i-1}, t_{i}\right) f\left(t_{i}\right)-\sum_{1}^{n} V_{1}\left(t_{i-1}, t_{i}\right) f(y)\right\| \\
& \leqq \sum_{1}^{n}\left\|V_{2}\left(t_{i-1}, t_{i}\right) f\left(t_{i-1}\right)-V_{2}\left(t_{i-1}, t_{i}\right) f(x)\right\| \\
&+\sum_{1}^{n}\left\|V_{1}\left(t_{i-1}, t_{i}\right) f\left(t_{i}\right)-V_{1}\left(t_{i-1}, t_{i}\right) f(y)\right\| \\
& \leqq \sum_{1}^{n} \alpha_{2}\left(t_{i-1}, t_{i}\right) \beta\left(x, t_{i-1}\right)+\sum_{1}^{n} \alpha_{1}\left(t_{i-1}, t_{i}\right) \beta\left(t_{i}, 3\right) \\
&= \alpha_{2}(x, y) \beta(x, c)-\sum_{t} \alpha_{2} \beta(\quad, c)[L]+\sum_{t} \alpha_{1} \beta(\quad, c)[R] \\
&-\alpha_{1}(x, y) \beta(y, c) .
\end{aligned}
$$

Let $h(x, y)=\alpha_{2}(x, y) \beta(x, c)-{ }_{x} \sum^{y} \alpha_{2} \beta(, c)[L]$

$$
+{ }_{x} \sum^{y} \alpha_{1} \beta(, c)[R]-\alpha_{1}(x, y) \beta(y, c) .
$$

Since ${ }_{x} \Sigma^{y} \alpha_{2} \beta(, c)[L]$ and ${ }_{x} \Sigma^{y} \alpha_{1} \beta(, c)[R]$ exist for every $\{x, y\}$ in $S X S$ (as in [7, p. 629]) and each is real valued then by a theorem of Kolmogoroff's [6, p. 668] ${ }_{a} \Sigma^{b} h=0$ for all $\{a, b\}$ in $S X S$ and the proof is complete. 
Lemma 2. Suppose

(1) each of $V_{1}$ and $V_{2}$ is in $\mathscr{O A}$, each of $\alpha_{1}$ and $\alpha_{2}$ is in $\mathscr{O A}+$ and there is a number $a<1$ such that for each $\{x, y\}$ in $S X S$ and $\{P, Q\}$ in $G X G$

$$
\begin{aligned}
& \left\|V_{1}(x, y) P-V_{1}(x, y) Q\right\| \leqq \alpha_{1}(x, y)\|P-Q\| \text { and } \\
& \left\|V_{2}(x, y) P-V_{2}(x, y) Q\right\| \leqq \alpha_{2}(x, y)\|P-Q\| \leqq a\|P-Q\| ;
\end{aligned}
$$

(2) $C$ is a function from $S X S$ to $G$ such that for each $\{x, y\}$ in $S X S$, ${ }_{x} \Sigma^{y}\|C\|=0$

(3) ${ }_{x} \Pi^{y}\left[1-V_{2}\right]^{-1}\left[1+V_{1}\right] P$ exists for every $\{x, y, P\}$ in $S X S X G$; and

(4) $A(x, y) P=\left[1-V_{2}(x, y)\right]^{-1}\left\{\left[1+V_{1}(x, y)\right] P+C(x, y)\right\}$ for each $\{x, y, P\}$ in $S X S X G$.

Conclusion. ${ }_{x} \Pi^{y}\left[1-V_{2}\right]^{-1}\left[1+V_{1}\right] P={ }_{x} \Pi^{y} A P$ for every $\{x, y, P\}$ in $S X S X G$.

Proof. First note from Theorem 3.3 that $\|\left[1-V_{2}(x, y)\right]^{-1}\left\{\left[1+V_{1}(x, y)\right] P+C(x, y)\right\}$

$$
-\left[1-V_{2}(x, y)\right]^{-1}\left[1+V_{1}(x, y)\right] P \|
$$

$\leqq\left[1-\alpha_{2}(x, y)\right]^{-1}\|C(x, y)\|$ for each $\{x, y, P\}$ in $S X S X G$. Let $\{x, y, P\}$ be in $S X S X G$ and $t$ be an $\mathcal{O}$-subdivision of $\{x, y\}$. Using Lemma 1.2 [7, p. 623], $\quad\left\|\Pi_{t} A P-\Pi_{t}\left[1-V_{2}\right]^{-1}\left[1+V_{1}\right] P\right\| \leqq \Pi_{t}\left[1-\alpha_{2}\right]^{-1}\left\{\left[1+\alpha_{1}\right]+\|C\|\right\}-$ $\Pi_{t}\left[1-\alpha_{2}\right]^{-1}\left[1+\alpha_{1}\right]$; from Theorem $3.2[2$, p. 300] and our hypothesis there is a number $b$ such that the difference between these last two products does not exceed $b \Sigma_{t}\|C\|$, which completes our proof.

THEOREM 4.1. Suppose

(1) each of $V_{1}$ and $V_{2}$ is in $\mathscr{O A}$,

(2) $K$ is in $\Phi\left(V_{1}\right)$ and $M$ is in $\Phi\left(V_{2}\right)$,

(3) $f$ is a function from $S$ to $G$ that is bounded on each O-interval of $S$, and

(4) for each $\{x, y\}$ in $S X S \int_{x}^{y}\left(V_{1} f[R]+V_{2} f[L]\right)$ exists.

Conclusion. For each $\{x, y\}$ in $S X S$

$$
\int_{x}^{y}(K f[R]+M f[L])=\int_{x}^{y}\left(V_{1} f[R]+V_{2} f[L]\right) .
$$

Proof. Let each of $h$ and $k$ be a function from $S X S$ to the real numbers such that for each $\{x, y, P\}$ in $S X S X G,{ }_{x} \Sigma^{y} k=0$, $\left\|K(x, y) P-V_{1}(x, y) P\right\| \leqq k(x, y)\|P\|,{ }_{x} \Sigma^{y} h=0$, and

$$
\left\|M(x, y) P-V_{2}(x, y) P\right\| \leqq h(x, y)\|P\| .
$$


Pick $\{x, y\}$ in $S X S$ and a number $b$ such that if $\{x, z, y\}$ is an $\mathcal{O}$-subdivision of $\{x, y\}$ then $\|f(z)\| \leqq b$. Let $\left\{t_{i}\right\}_{0}^{n}$ be an $\mathcal{O}$-subdivision of $\{x, y\}$; then

$$
\begin{aligned}
\| & \sum_{1}^{n}\left\{K\left(t_{i-1}, t_{i}\right) f\left(t_{i}\right)+M\left(t_{i-1}, t_{i}\right) f\left(t_{i-1}\right)\right\} \\
& -\sum_{1}^{n}\left\{V_{1}\left(t_{i-1}, t_{i}\right) f\left(t_{i}\right)+V_{2}\left(t_{i-1}, t_{i}\right) f\left(t_{i-1}\right)\right\} \| \\
\leqq & \sum_{1}^{n}\left\|K\left(t_{i-1}, t_{i}\right) f\left(t_{i}\right)-V_{1}\left(t_{i-1}, t_{i}\right) f\left(t_{i}\right)\right\| \\
& +\sum_{1}^{n}\left\|M\left(t_{i-1}, t_{i}\right) f\left(t_{i-1}\right)-V_{2}\left(t_{i-1}, t_{i}\right) f\left(t_{i-1}\right)\right\| \\
\leqq & \sum_{1}^{n} k\left(t_{i-1}, t_{i}\right)\left\|f\left(t_{i}\right)\right\|+\sum_{1}^{n} h\left(t_{i-1}, t_{i}\right)\left\|f\left(t_{i-1}\right)\right\| \\
\leqq & b\left\{\sum_{1}^{n} k\left(t_{i-1}, t_{i}\right)+\sum_{1}^{n} h\left(t_{i-1}, t_{i}\right)\right\} .
\end{aligned}
$$

REMARK. The construction of the proof of the next theorem is similar to that of Theorem 5.1 [2, p. 310].

\section{Theorem 4.2. Suppose}

(1) each of $V_{1}$ and $V_{2}$ is in $\mathscr{O A}$ and each of $\alpha_{1}$ and $\alpha_{2}$ is in $\mathscr{O A}+$ such that for each $\{x, y\}$ in $S X S$ and $\left\{Q_{1}, Q_{2}\right\}$ in $G X G$

$$
\begin{aligned}
& \left\|V_{1}(x, y) Q_{1}-V_{1}(x, y) Q_{2}\right\| \leqq \alpha_{1}(x, y)\left\|Q_{1}-Q_{2}\right\| \text { and } \\
& \left\|V_{2}(x, y) Q_{1}-V_{2}(x, y) Q_{2}\right\| \leqq \alpha_{2}(x, y)\left\|Q_{1}-Q_{2}\right\| ;
\end{aligned}
$$

(2) $K$ is in $\Phi\left(V_{1}\right)$ and $M$ is in $\Phi\left(V_{2}\right)$ and each of $h$ and $k$ is a function from $S X S$ to the real numbers such that for each $\{x, y, Q\}$ in $S X S X G,{ }_{x} \Sigma^{y} k=0,\left\|V_{1}(x, y) Q-K(x, y) Q\right\| \leqq k(x, y)\|Q\|,{ }_{x} \Sigma^{y} h=0$, $\left\|V_{2}(x, y) Q-M(x, y) Q\right\| \leqq h(x, y)\|Q\|$ and there is a number $a<1$ such that $\alpha_{2}(s, t)+h(s, t)<a$ for all $\{s, t\}$ in $S X S$;

(3) $\{c, P\}$ is in $S X G$.

Conclusion. The following statements are equivalent:

(1) $f$ is in $\mathscr{F}(x, P)$ and $f(x)=P+\int_{x}^{c}(K f[R]+M f[L])$ for each $x$ in $S$;

(2) $f(x)={ }_{x} \Pi^{c}[1-M]^{-1}[1+K] P$ for each $x$ in $S$; and

(3) if for each $\{a, b, Q\}$ in $S X S X G$ 


$$
V(a, b) Q={ }_{a} \sum^{b}\left\{[1-M]^{-1}[1+K]-1\right\} Q
$$

then $f(x)=x \Pi^{c}[1+V] P$ for each $x$ in $S$.

Proof. $(1 \rightarrow 2)$ : If $\{x, y, c\}$ is an $\mathcal{O}$-subdivision of $\{x, c\}$ then by Theorem 4.1

$$
\begin{aligned}
f(x) & =P+\int_{x}^{c}(K f[R]+M f[L])=P+\int_{x}^{c}\left(V_{1} f[R]+V_{2} f[L]\right) \\
& =f(y)+\int_{x}^{y}\left(V_{1} f[R]+V_{2} f[L]\right) .
\end{aligned}
$$

Hence if $\left\{t_{j}\right\}_{0}^{n}$ is an $\mathcal{O}$-subdivision of $\{x, c\}$ and $j$ is an integer in $[1, n]$ then

$$
\begin{aligned}
& f\left(t_{j-1}\right)-f\left(t_{j}\right)=\int_{t_{j-1}}^{t_{j}}\left(V_{1} f[R]+V_{2} f[L]\right) \text { and } \\
& f\left(t_{j-1}\right)=f\left(t_{j}\right)+V_{1}\left(t_{j-1}, t_{j}\right) f\left(t_{j}\right)+V_{2}\left(t_{j-1}, t_{j}\right) f\left(t_{j-1}\right) \\
& +C\left(t_{j-1}, t_{j}\right) \\
& \text { where } C\left(t_{j-1}, t_{j}\right)=\int_{t_{i-1}}^{t_{j}}\left(V_{1} f[R]+V_{2} f[L]\right)-V_{1}\left(t_{j-1}, t_{j}\right) f\left(t_{j}\right) \\
& -V_{2}\left(t_{j-1}, t_{j}\right) f\left(t_{j-1}\right) \text {. } \\
& {\left[1-V_{2}\left(t_{j-1}, t_{j}\right)\right] f\left(t_{j-1}\right)=\left[1+V_{1}\left(t_{j-1}, t_{j}\right)\right] f\left(t_{j}\right)+C\left(t_{j-1}, t_{j}\right) .} \\
& f\left(t_{1-1}\right)=\left[1-V_{2}\left(t_{j-1}, t_{j}\right)\right]^{-1}\left\{\left[1+V_{1}\left(t_{j-1}, t_{j}\right)\right] f\left(t_{j}\right)+C\left(t_{j-1}, t_{j}\right)\right\} .
\end{aligned}
$$

Let $A(x, y) Q=\left[1-V_{2}(x, y)\right]^{-1}\left\{\left[1+V_{1}(x, y)\right] Q+C(x, y)\right\}$. By iteration $j=n, n-1, n-2, \cdots, 1$, in order, we obtain

$$
f\left(t_{0}\right)=\prod_{j=1}^{n} A\left(t_{j-1}, t_{j}\right) f\left(t_{n}\right)
$$

Using our Lemmas 1 and 2 and Theorem 3.3

$$
f(x)={ }_{x} \prod^{c}\left[1-V_{2}\right]^{-1}\left[1+V_{1}\right] P={ }_{x} \prod^{c}[1-M]^{-1}[1+K] P .
$$

$(2 \rightarrow 1):$ If $\{x, c\}$ is in $S X S$ and $\left\{t_{t}\right\}_{0}^{n}$ is an $\mathcal{O}^{\prime}$-subdivision of $\{x, c\}$ and $i$ is an integer in $[1, n]$ then from Theorem 3.3

$$
\begin{aligned}
f\left(t_{t-1}\right) & ={ }_{t_{i-1}} \prod^{t_{i}}[1-M]^{-1}[1+K] f\left(t_{i}\right) \\
& ={ }_{t_{i-1}} \prod^{t_{i}}\left[1-V_{2}\right]^{-1}\left[1+V_{1}\right] f\left(t_{i}\right)
\end{aligned}
$$




$$
\begin{aligned}
= & {\left[1-V_{2}\left(t_{i-1}, t_{i}\right)\right]^{-1}\left[1+V_{1}\left(t_{i-1}, t_{i}\right)\right] f\left(t_{i}\right) } \\
& +D\left(t_{i-1}, t_{i}\right) f\left(t_{i}\right)
\end{aligned}
$$

$$
\text { where } \begin{aligned}
D\left(t_{i-1}, t_{i}\right)= & { }_{t_{i-1}} \prod^{t_{i}}\left[1-V_{2}\right]^{-1}\left[1+V_{1}\right] \\
& -\left[1-V_{2}\left(t_{i-1}, t_{i}\right)\right]^{-1}\left[1+V_{1}\left(t_{i-1}, t_{i}\right)\right]
\end{aligned}
$$$$
\left[1-V_{2}\left(t_{i-1}, t_{i}\right)\right]\left[f\left(t_{i-1}\right)-D\left(t_{i-1}, t_{i}\right) f\left(t_{i}\right)\right]=\left[1+V_{1}\left(t_{i-1}, t_{i}\right)\right] f\left(t_{i}\right)
$$$$
f\left(t_{i-1}\right)-f\left(t_{i}\right)=V_{1}\left(t_{i-1}, t_{i}\right) f\left(t_{i}\right)+V_{2}\left(t_{i-1}, t_{i}\right)\left[f\left(t_{i-1}\right)\right.
$$$$
\left.-D\left(t_{i-1}, t_{i}\right) f\left(t_{i}\right)\right]+D\left(t_{i-1}, t_{i}\right) f\left(t_{i}\right)
$$

$$
f(x)=f(c)+\int_{x}^{c}\left\{V_{1} f[R]+V_{2}(f[L]+D f[R])\right\}+{ }_{x} \Sigma^{c} D f[R]
$$

but $\int_{x}^{c} V_{2}[f[L]+D f[R]]+{ }_{x} \sum^{c} D f[R]=\int_{x}^{c} V_{2} f[L]$ because

$$
\begin{aligned}
& \| \sum_{1}^{n} V_{2}\left(t_{i-1}, t_{i}\right)\left[f\left(t_{i-1}\right)-D\left(t_{i-1}, t_{i}\right) f\left(t_{i}\right)\right] \\
& \quad+\sum_{1}^{n} D\left(t_{i-1}, t_{i}\right) f\left(t_{i}\right)-\sum_{1}^{n} V_{2}\left(t_{i-1}, t_{i}\right) f\left(t_{i-1}\right) \| \\
& \leqq \sum_{1}^{n} \alpha_{2}\left(t_{i-1}, t_{i}\right)\left\|D\left(t_{i-1}, t_{i}\right) f\left(t_{i}\right)\right\|+\sum_{1}^{n}\left\|D\left(t_{i-1}, t_{i}\right) f\left(t_{i}\right)\right\| \\
& \leqq\left\{1+\alpha_{2}(x, c)\right\} \sum_{1}^{n}\left\|D\left(t_{i-1}, t_{i}\right) f\left(t_{i}\right)\right\| \\
& \leqq \\
&
\end{aligned}
$$

where $d(a, b)={ }_{a} \Pi^{b}\left[1-\alpha_{2}\right]^{-1}\left[1+\alpha_{1}\right]-\left[1-\alpha_{2}(a, b)\right]^{-1}\left[1+\alpha_{1}(a, b)\right]$ for each $\{a, b\}$ in $S X S$. The preceding inequality follows from the proof of Theorem 3.2, and it follows from Theorem 3.0 and [6, p. 668] that ${ }_{a} \Sigma^{b} d=0$ for each $\{a, b\}$ in $S X S$.

Hence $f(x)=P+\int_{x}^{c}\left(V_{1} f[R]+V_{2} f[L]\right)=P+\int_{x}^{c}(K f[R]+M f[L])$.

It follows from Theorems 3.2 and 3.3 that (3) is equivalent to (2) and the proof is complete. 
REMARK. From the foregoing argument it is evident that each of the following statements is equivalent to those in the conclusion of the preceding theorem:

(4) $f$ is in $\mathscr{F}(c, P)$ and

$$
f(x)=P+\int_{x}^{c}\left(V_{1} f[R]+V_{2} f[L]\right)
$$

for each $x$ in $S$; and

(5) $f(x)={ }_{x} \Pi^{c}\left[1-V_{2}\right]^{-1}\left[1+V_{1}\right] P$ for each $x$ in $S$.

THEOREM 4.3. Suppose

(1) each of $V_{1}$ and $V_{2}$ is in $O \mathscr{A}$ and each of $\alpha_{1}$ and $\alpha_{2}$ is in $\mathscr{O A}{ }^{+}$such that for each $\{x, y\}$ in $S X S$ and $\left\{Q_{1}, Q_{2}\right\}$ in $G X G$

$$
\begin{aligned}
& \left\|V_{1}(x, y) Q_{1}-V_{1}(x, y) Q_{2}\right\| \leqq \alpha_{1}(x, y)\left\|Q_{1}-Q_{2}\right\| \text { and } \\
& \left\|V_{2}(x, y) Q_{1}-V_{2}(x, y) Q_{2}\right\| \leqq \alpha_{2}(x, y)\left\|Q_{1}-Q_{2}\right\|
\end{aligned}
$$

(2) $K$ is in $\Phi\left(V_{1}\right)$ and $M$ is in $\Phi\left(V_{2}\right)$ and each of $h$ and $k$ is a function from $S X S$ to the real numbers such that for each $\{x, y, Q\}$ in $S X S X G,{ }_{x} \Sigma^{y} k=0$,

$$
\begin{aligned}
& \left\|V_{1}(x, y) Q-K(x, y) Q\right\| \leqq k(x, y)\|Q\|,{ }_{x} \sum^{y} h=0, \\
& \left\|V_{2}(x, y) Q-M(x, y) Q\right\| \leqq h(x, y)\|Q\|,
\end{aligned}
$$

and there is a number $a<1$ such that $\alpha_{1}(s, t)+k(s, t) \leqq$ a for all $\{s, t\}$ in $S X S$;

(3) $K^{\prime}(y, x) Q=K(x, y) Q, M^{\prime}(y, x) Q=M(x, y) Q$ for each $\{x, y, Q\}$ in $S X S X G$;

(4) $\{c, P\}$ is in $S X G$.

Conclusion. The following statements are equivalent:

(1) $f$ is in $\mathscr{F}(c, P)$ and $f(x)=P+\int_{c}^{x}(K f[R]+M f[L])$ for $x$ in $S$;

(2) $f(x)={ }_{x} \Pi^{c}\left[1-K^{\prime}\right]^{-1}\left[1+M^{\prime}\right] P$ for each $x$ in $S$; and

(3) if $V(a, b) Q={ }_{a} \Sigma^{b}\left\{\left[1-K^{\prime}\right]^{-1}\left[1+M^{\prime}\right]-1\right\} Q$ for each $\{a, b, Q\}$ in $S X S X G$, then

$$
f(x)={ }_{x} \Pi^{c}[1+V] P \text { for each } x \text { in } S .
$$

The proof of this theorem, except for minor algebraic manipulations, is the same as the proof of the previous theorem. 
5. A seemingly more general integral equation. In [7, pp. 632-633] Mac Nerney showed that the theory he developed in solving an integral equation of the form $f(x)=P+(R) \int_{x}^{c} V f$ could be used to solve a seemingly more general equation of the form

$$
f(x)=P_{1}+(R) \int_{x}^{c} V_{1} f+V_{2}(x, c) P_{2} .
$$

We repeat that procedure here by using the theory developed in the preceding section to solve an equation of the form

$$
f(x)=P_{1}+\int_{c}^{x}(K f[R]+M f[L])+V(x, c) P_{2},
$$

and the solution of this equation in the purely linear case is shown to include the solutions Helton obtained in Theorems 5.1-5.4 [2, pp. 310-314].

Let $\{G X G,+,\|\|\}, \mathscr{O A} "$ and $\mathscr{O} \mathcal{M}^{\prime \prime}$ be defined as in [7, p. 632]. Let $\Phi^{\prime \prime}$ and $\psi^{\prime \prime}$ be the mappings corresponding to the mappings $\Phi$ and $\psi$. The following theorem is a reinterpretation of Theorem 4.3. We will not state the corresponding reinterpretation of Theorem 4.2.

THEOREM 5. Assume the hypothesis of Theorem 4.3 with $K$ and $M$ as defined there. Let $P$ be in $G X G, V$ be in $O A A$ and each of $K^{\prime \prime}$ and $M^{\prime \prime}$ be in $\Phi^{\prime \prime}\left(\mathscr{O A A}^{\prime \prime}\right)$ such that

$$
\begin{aligned}
& K^{\prime \prime}(x, y) Q=\left\{K(y, x) Q_{1}, 0\right\} \text { and } \\
& M^{\prime \prime}(x, y) Q=\left\{M(y, x) Q_{1}+V(y, x) Q_{2}, 0\right\}
\end{aligned}
$$

for each $\{x, y\}$ in $S X S$ and $Q$ in $G X G$. If $u$ is a function from $S$ to $G$, the following are equivalent :

(1) $\left\{u(x), P_{2}\right\}={ }_{x} \Pi^{c}\left[1-K^{\prime \prime}\right]^{-1}\left[1+M^{\prime \prime}\right] P$ for each $x$ in $S$, and

(2) $u$ is in $\mathscr{F}\left(c, P_{1}\right)$ such that for each $x$ in $S$

$$
u(x)=P_{1}+\int_{c}^{x}(K u[R]+M u[L])+V(x, c) P_{2}
$$

The next corollary shows that in the purely linear case this theorem includes the solutions Helton obtained in Theorems 5.1-5.4 [2, pp. 310-314].

Let $\{N,+, \cdot, \mid\}$ be a complete normed ring.

Corollary. Suppose

(1) each of $K$ and $M$ is a function from $S X S$ to $N$ that is in the common part of $\mathbf{O A}^{0}$ and $\mathbf{O B}^{0}[2$, p. 299]; 
(2) there exists a number $a<1$ such that for each $\{x, y\}$ in $S X S$

$$
\left|K(x, y)-{ }_{x} \sum^{y} K\right|+{ }_{x} \sum^{y}|K| \leqq a \text { and }
$$

$M^{\prime}(x, y)=M(y, x)$ and $K^{\prime}(x, y)=K(y, x)$; and

(3) $c$ is in $S$ and each of $f$ and $h$ is a function from $S$ to $N$ such that $f(c)=h(c)$ and $d h$ is in $O B^{0}$.

Conclusions.

(1) The following two statements are equivalent:

(a) df is in $O B^{0}$ and

$f(x)=h(x)+\int_{c}^{x}(f[R] K+f[L] M)$ for each $x$ in $S ;$ and

(b) $f(x)=f(c)_{c} \Pi^{x}[1+M][1-K]^{-1}$

$$
+\int_{c}^{x}(d h)_{t} \Pi^{x}[1+M)[1-K]^{-1}[R]
$$

for each $x$ in $S$.

(2) The following two statements are equivalent:

(a) df is in $O B^{\circ}$ and

$$
\begin{gathered}
f(x)=h(x)+\int_{c}^{x}(K f[R]+M f[L]) \text { for each } x \text { in } S ; \text { and } \\
\text { (b) } f(x)={ }_{x} \Pi^{c}\left[1-K^{\prime}\right]^{-1}\left[1+M^{\prime}\right] f(c) \\
\quad+\int_{c}^{x}{ }_{x} \Pi^{t}\left[1-K^{\prime}\right]^{-1}\left[1+M^{\prime}\right][R] d h
\end{gathered}
$$

for each $x$ in $S$.

(3) The following two statements are equivalent:

(a) df is in $O B^{0}$ and

$$
\begin{array}{r}
f(x)=h(x)+\int_{c}^{x}(K f[R]+f[L] M) \text { for each } x \text { in } S \text {; and } \\
\text { (b) } \begin{aligned}
f(x)= & { }_{x} \Pi^{c}\left[1-K^{\prime}\right]^{-1} f(c)_{c} \Pi^{x}[1+M] \\
& +\int_{c}^{x}{ }_{x} \Pi^{t}\left[1-K^{\prime}\right]^{-1}[R](d h)_{t} \Pi^{x}[1+M][R]
\end{aligned}
\end{array}
$$

for each $x$ in $S$.

(4) The following two statements are equivalent: 
(a) df is in $O B^{0}$ and $f(x)=h(x)+\int_{c}^{x}(f[R] K+M f[L])$ for each $x$ in $S$; and

(b) $f(x)={ }_{x} \Pi^{c}\left[1+M^{\prime}\right] f(c)_{c} \Pi^{x}[1-K]^{-1}$

$$
+\int_{c}^{x}{ }_{x} \Pi^{t}\left[1+M^{\prime}\right][R](d h)_{t} \Pi^{x}[1-K]^{-1}[R]
$$

for each $x$ in $S$.

Indication of proof.

(1) For each $\{x, y\}$ in $S X S$ and $Q$ in $N X N$

$$
\begin{aligned}
& K^{\prime \prime}(x, y)[Q]=\left\{Q_{1} \cdot K(y, x), 0\right\} \text { and } \\
& M^{\prime \prime}(x, y)[Q]=\left\{Q_{1} \cdot M(y, x)-d h(x, y) Q_{2}, 0\right\}
\end{aligned}
$$

Let $P$ be in NXN such that $P_{1}=h(c)$ and $P_{2}=1$;

$$
\begin{aligned}
h(x)+ & \int_{c}^{x}(f[R] K+f[L] M) \\
& =P_{1}+\int_{x}^{c}\left(K^{\prime \prime} f[R]+M^{\prime \prime} f[L]\right)+(-d h)(c, x) \cdot P_{2},
\end{aligned}
$$

and for each $\mathcal{O}$-subdivision $\left\{t_{j}\right\}_{0}^{n}$ of $\{x, c\}$

$$
\begin{aligned}
& \prod_{j=1}^{n}[1-\left.K^{\prime \prime}\left(t_{j-1}, t_{j}\right)\right]^{-1}\left[1+M^{\prime \prime}\left(t_{j-1}, t_{j}\right)\right] P \\
&=\left\{f(c) \prod_{j=1}^{n}\left[1+M\left(t_{n-j+1}, t_{n-j}\right)\right]\left[1-K\left(t_{n-j+1}, t_{n-j}\right)\right]^{-1}\right. \\
&+\sum_{j=1}^{n} d h\left(t_{n-j}, t_{n-j+1}\right) \prod_{q=1}^{n-j}\left[1+M\left(t_{n-q+1}, t_{n-q}\right)\right] \\
&\left.\cdot\left[1-K\left(t_{n-q+1}, t_{n-q}\right)\right]^{-1}, P_{2}\right\} \cdot \\
& \text { (2) } \quad K^{\prime \prime}(x, y)[Q]=\left\{K^{\prime}(x, y) \cdot Q_{1}, 0\right\} \text { and } \\
& M^{\prime \prime}(x, y)[Q]=\left\{M^{\prime}(x, y) \cdot Q_{1}-d h(x, y) \cdot Q_{2}, 0\right\}
\end{aligned}
$$

for each $\{x, y\}$ in $S X S$ and $Q$ in $N X N$.

(3) $K^{\prime \prime}(x, y)[Q]=\left\{K^{\prime}(x, y) \cdot Q_{1}, 0\right\} \quad$ and

$$
M^{\prime \prime}(x, y)[Q]=\left\{Q_{1} \cdot M(y, x)-d h(x, y) \cdot Q_{2}, 0\right\}
$$

for each $\{x, y\}$ in $S X S$ and $Q$ in $N X N$. 
(4) $K^{\prime \prime}(x, y)[Q]=\left\{Q_{1} \cdot K(y, x), 0\right\}$ and

$$
M^{\prime \prime}(x, y)[Q]=\left\{M^{\prime}(x, y) \cdot Q_{1}-d h(x, y) \cdot Q_{2}, 0\right\}
$$

for each $\{x, y\}$ in $S \times S$ and $Q$ in $N \times N$.

Acknowledgment. The author would like to thank Professor J. S. Mac Nerney for his many helpful suggestions in the writing of this paper.

\section{REFERENCES}

1. W. L. Gibson, Stieltjes and Stieltjes-Volterra integral equations, Ph. D. Dissertation, University of Houston (1974).

2. B. W. Helton, Integral equations and product integrals, Pacific J. of Math., 16 (1966), 297-322.

3. A product integral representation for a Gronwall inequality, Proc. Amer. Math. Soc., 23, No. 3 (1969), 493-500.

4. J. C. Helton, Product integrals and inverses in normed rings, Pacific J. of Math., 51 (1974), $155-166$.

5. J. V. Herod, Coalescence of solutions for nonlinear Stieltjes equations, J. Reine Angew. Math., 252 (1972), 187-194.

6. A. Kolmogoroff, Untersuchungen über den Integralbegriff, Math. Ann., 103 (1930), 654-696.

7. J. S. Mac Nerney, A nonlinear integral operation, Illinois J. Math., 8 (1964), 621-638.

8. J. W. Neuberger, Toward a characterization of the identity component of rings and near-rings of continuous transformations, J. Reine Angew. Math., 238 (1969), 100-104.

Received May 29, 1974 and in revised form September 9, 1974. 
D. E. Bennett, Strongly unicoherent continua ............................. 1

Walter R. Bloom, Sets of p-spectral synthesis ................................ 7

R. T. Bumby and D. E. Dobbs, Amitsur cohomology of quadratic extensions: Formulas and number-theoretic examples ................. 21

W. W. Comfort, Compactness-like properties for generalized weak topological sums

D. R. Dunninger and J. Locker, Monotone operators and nonlinear biharmonic boundary value problems ...

T. S. Erickson, W. S. Martindale, 3rd and J. M. Osborn, Prime nonassociative algebras

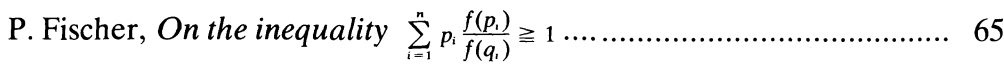

G. Fox and P. Morales, Compact subsets of a Tychonoff set ............... 75

R. Gilmer and J. F. Hoffmann, A characterization of Prüfer domains in terms of polynomials ......................................................... 81

L. C. Glaser, On tame Cantor sets in spheres having the same projection in each direction ......................................................... 87

Z. Goseki, On semigroups in which $X=X Y X=X Z X$ if and only if

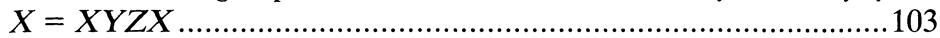

E. Grosswald, Rational valued series of exponentials and divisor

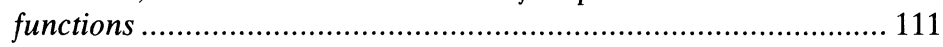

D. Handelman, Strongly semiprime rings ...................................... 115

J. N. Henry and D. C. Taylor, The $\bar{\beta}$ topology for $w^{*}$-algebras ............. 123

M. J. Hodel, Enumeration of weighted p-line arrays .......................... 141

S. K. Jain and S. Singh, Rings with quasiprojective left ideals .............. 169

S. Jeyaratnam, The diophantine equation $Y(Y+m)(Y+2 m) \times$

$$
(Y+3 m)=2 X(X+m)(X+2 m)(X+3 m) \ldots \ldots \ldots \ldots \ldots \ldots \ldots . . .183
$$

$\mathrm{R}$. Kane, On loop spaces without $p$ torsion .........................................189

Alvin J. Kay, Nonlinear integral equations and product integrals ..........203

A. S. Kechris, Countable ordinals and the analytic hierarchy, I ...........223

Ka-Sing Lau, A representation theorem for isometries of $C(X, E) \ldots \ldots . .229$

I. Madsen, On the action of the Dyer-Lashof algebra in $H_{*}(G)$..........235

R. C. Metzler, Positive linear functions, integration, and Choquet's theorem ........................................................................................ 277

A. Nobile, Some properties of the Nash blowing-up ............................297

G. E. Petersen and G. V. Welland, Plessner's theorem for Riesz conjugates 


\section{Pacific Journal of Mathematics}

\section{Vol. 60, No. $1 \quad$ September, 1975}

Donald Earl Bennett, Strongly unicoherent continua ................ 1

Walter Russell Bloom, Sets of p-spectral synthesis ................ 7

Richard Thomas Bumby and David Earl Dobbs, Amitsur cohomology of

quadratic extensions: formulas and number-theoretic examples .......

W. Wistar (William) Comfort, Compactness-like properties for generalized

weak topological sums ...................................

Dennis Robert Dunninger and John Stewart Locker, Monotone operators

and nonlinear biharmonic boundary value problems ..............

Theodore Erickson, Wallace Smith Martindale, III and J. Marshall Osborn,

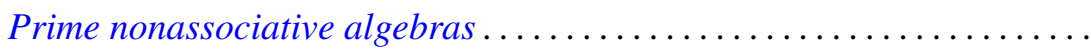

Pál Fischer, On the inequality $\sum_{i=0}^{n}\left[f\left(p_{i}\right) / f\left(q_{i}\right)\right] p_{i} \geq i \ldots \ldots \ldots \ldots \ldots$

Geoffrey Fox and Pedro Morales, Compact subsets of a Tychonoff set.......

Robert William Gilmer, Jr. and Joseph F. Hoffmann, A characterization of

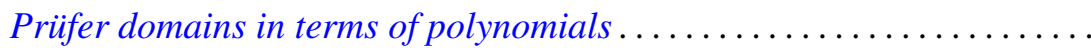

Leslie C. Glaser, On tame Cantor sets in spheres having the same projection

in each direction . ...................................

Zensiro Goseki, On semigroups in which $x=x y x=x z x$ if and only if

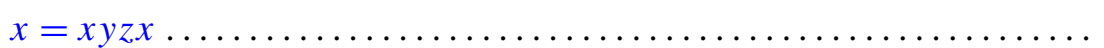

Emil Grosswald, Rational valued series of exponentials and divisor

functions.

David E. Handelman, Strongly semiprime rings

Jackson Neal Henry and Donald Curtis Taylor, The $\bar{\beta}$ topology for

$W^{*}$-algebras

Margaret Jones Hodel, Enumeration of weighted p-line arrays ...

Surender Kumar Jain and Surjeet Singh, Rings with quasi-projective left

ideals.

S. Jeyaratnam, The Diophantine equation

$$
Y(Y+m)(Y+2 m)(Y+3 m)=2 X(X+m)(X+2 m)(X+3 m) \ldots
$$

Richard Michael Kane, On loop spaces without $p$ torsion

Alvin John Kay, Nonlinear integral equations and product integrals ...

Alexander S. Kechris, Countable ordinals and the analytical hierarchy.

$I$.

Ka-Sing Lau, A representation theorem for isometries of $C(X, E)$

Ib Henning Madsen, On the action of the Dyer-Lashof algebra in $H_{*}(G)$

Richard C. Metzler, Positive linear functions, integration, and Choquet's

theorem.

Augusto Nobile, Some properties of the Nash blowing-up

Gerald E. Peterson and Grant Welland, Plessner's theorem for Riesz. 